\title{
Direct Fixation of C1 Jefferson Fracture Using C1 Lateral Mass Screws: A Case Report
}

\author{
ARAVIND KUMAR, MBBS, MRCS(ED), MRCS(GLAS), FRCS, FAMS,${ }^{1}{ }^{\text {JAMES ONGGO }}{ }^{2}$ \\ LIM HAI FON, MBBS, MRCS, ${ }^{3}$ JACOB OH, MBBS, MMED, MRCS, FRCS ${ }^{4}$ \\ ${ }^{I}$ Ng Teng Fong Hospital, Department of Orthopaedics, Singapore, ${ }^{2}$ Monash University, Monash Medical School, Melbourne, Australia, ${ }^{3}$ Khoo Teck Puat \\ Hospital, Department of Orthopaedics, Singapore, ${ }^{4}$ Tan Tock Seng Hospital, Department of Orthopaedics, Singapore
}

\begin{abstract}
Background: Jefferson fractures are classically described as burst fractures of C1. Traditional surgical treatment of these unstable fractures is either occipito-cervical or posterior C1-C2 fusion, resulting in a significant loss of range of cervical neck movement. This case report discusses the fixation of a Jefferson fracture using C1 lateral mass screws to achieve surgical stabilization while preserving the range of movement. We aim to add to the body of evidence supporting this fixation method.

Methods: A 39-year-old male presented to our emergency department with an unstable Jefferson fracture caused by trauma. The various treatment options, such as cervical collar, HALO immobilization, occipito-cervical fusion, and atlanto-axial fusion and direct fixation of $\mathrm{C} 1$, were evaluated and discussed with the patient. Informed consent was obtained from the patient prior to writing this case report.

Results: At 3 months postoperation, the patient demonstrated near full range of movement in his neck. He had also resumed driving. By 6 months, the patient returned to work, and at 12 months, imaging confirmed union.

Conclusions: This case report adds to the body of evidence supporting the technique of $\mathrm{C} 1$ lateral mass screws for direct stable fixation of Jefferson fractures. This fixation method preserves the cervical spine range of motion with minimal functional restriction.
\end{abstract}

Cervical Spine

Keywords: Jefferson fractures, motion preserving, lateral mass screws, fracture fixation, fusion alternative, direct fixation, nonfusion, motion preserving, $\mathrm{C} 1$ fracture

\section{INTRODUCTION}

Jefferson fractures are burst fractures of $\mathrm{C} 1$ involving bilateral anterior and posterior arches, and they result from axial compression and hyperextension. They make up $2 \%$ to $13 \%$ of all cervical spine fractures. ${ }^{1}$ Stable fractures are often treated with a hard collar or HALO immobilization for 6 to 12 weeks. Surgical treatment of unstable fractures is, however, more controversial. Occipito-cervical or posterior atlanto-axial (C1-C2) fusion is often the surgery of choice. Unfortunately, fusion will result in significant loss of cervical spine range of movement. The following case report details an alternate surgical technique to treat unstable Jefferson fractures by means of direct fixation of $\mathrm{C} 1$ fracture with $\mathrm{C} 1$ lateral mass screws, aimed at preserving range of motion. Although described before, ${ }^{2,3}$ we hope that this case report adds to the body of evidence supporting it.

\section{CASE REPORT}

A 39-year-old male, with no medical comorbidities, presented to our emergency department complaining of neck pain for a week following a diving accident when on vacation overseas. He recounted impacting his head against the swimming pool floor. Initial radiographs taken in the emergency department overseas failed to diagnose the $\mathrm{C} 1$ fracture. He had no neurological deficits at any point. Further radiographs and computed tomographic (CT) scan revealed fractured anterior and posterior arches of $\mathrm{C} 1$ with centrifugal displacement of lateral masses (Figure 1). Carotid and vertebral artery CT angiograms were not indicated due to the subacute presentation and the absence of any clinical manifestations of vascular injury. A magnetic resonance scan later confirmed an intrasubstance tear of the transverse ligament (Figure 2). Treatment options discussed with him included HALO immobilization, occipito-cervical fusion, 


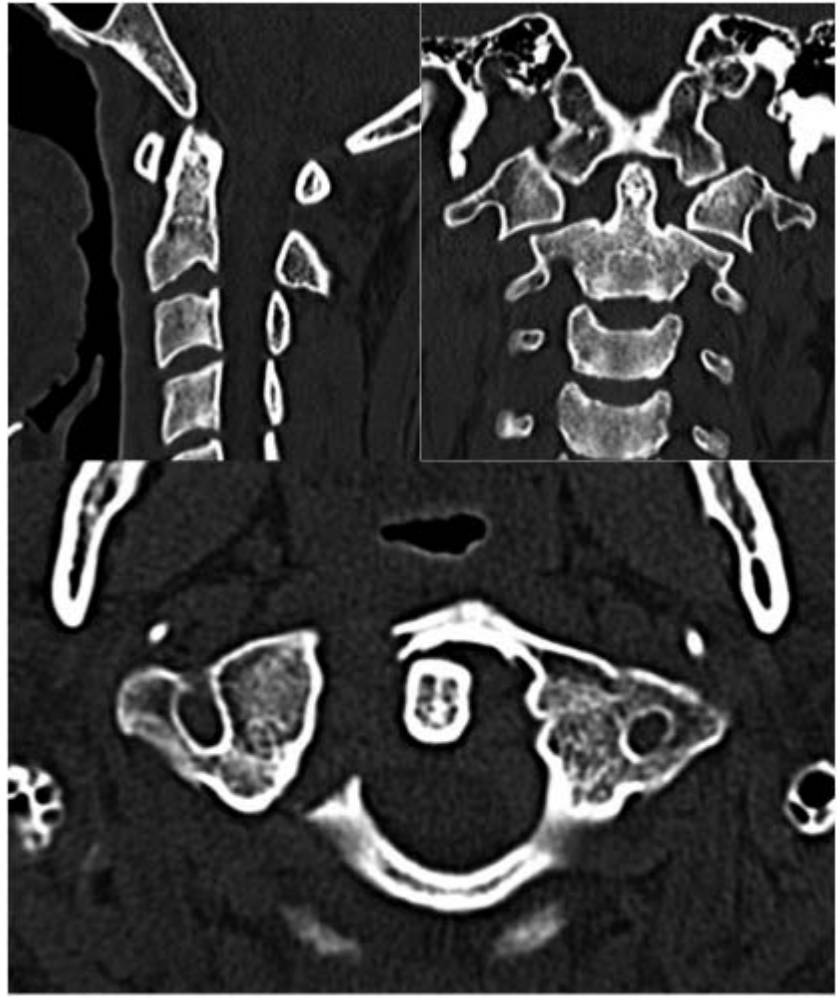

Figure 1. Preoperative computed tomographic scan showing the displaced fracture of the $\mathrm{C} 1$ vertebra with increased $\mathrm{C} 1$ lateral mass overhang over $\mathrm{C} 2$.

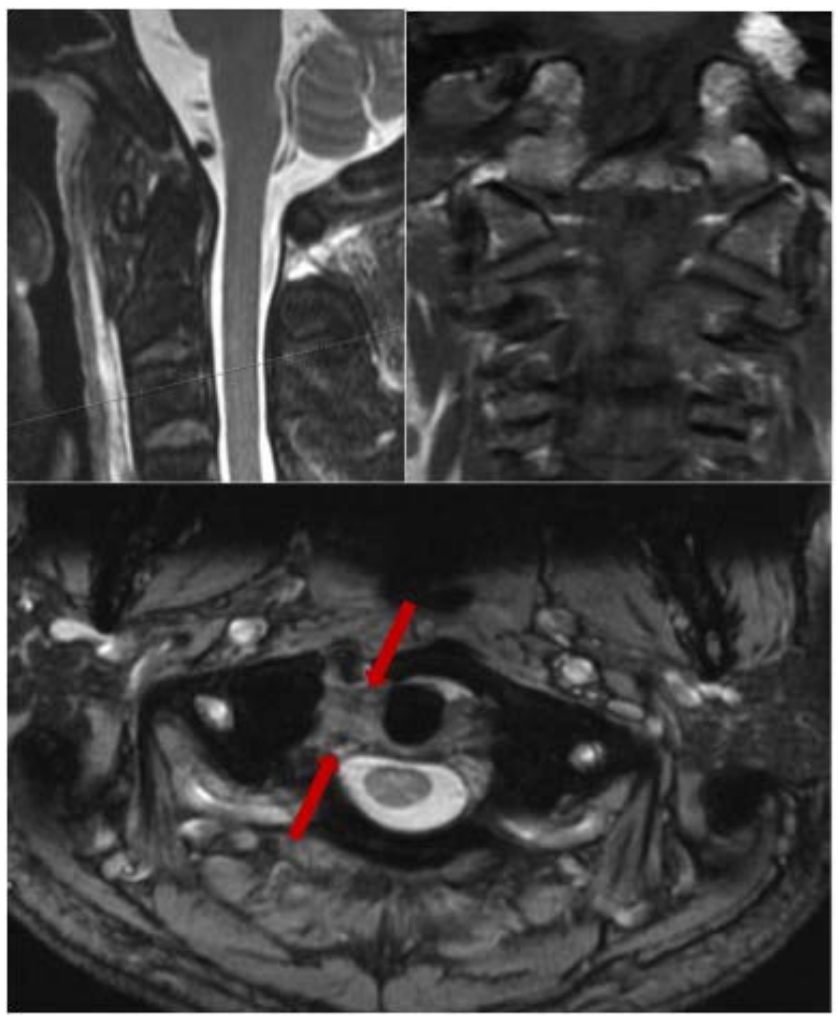

Figure 2. Preoperative magnetic resonance image (sagittal, coronal, and axial) showing an intrasubstance tear in the transverse ligament on the right side (between the red arrows)

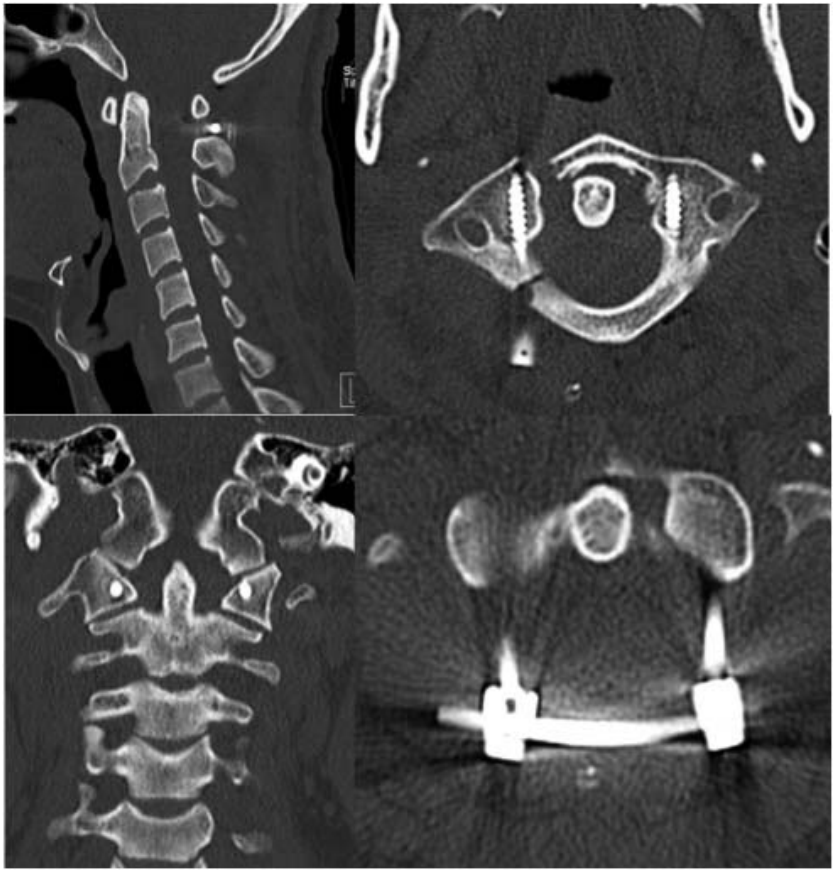

Figure 3. Postoperative computed tomographic scan showing the reduction of the posterior $\mathrm{C} 1$ ring and correction of the $\mathrm{C} 1$ lateral mass overhang.

C1-C2 fusion, or direct fixation with lateral mass screws. After careful consideration, the patient opted for direct fixation.

The surgery was performed in the prone position on a Jackson table with the head stabilized in a Mayfield headholder. Manual reduction was achieved by traction applied through the Mayfield clamp under fluoroscopic guidance. This was judged by the reduction of the overhang of $\mathrm{C} 1$ lateral masses. A midline incision was then made, and the paravertebral muscles were retracted to expose the $\mathrm{C} 1$ posterior arch. $\mathrm{C} 2$ nerve roots and vertebral arteries were identified and protected throughout to avoid iatrogenic injuries caused by misplacement or migration of instrumentation. The $\mathrm{C} 1$ lateral masses were meticulously exposed. The lower borders of overhanging $\mathrm{Cl}$ posterior arches were identified as entry points for lateral mass screws and burred to establish smooth entry points. Drilling through the lateral masses was done under II guidance. Partially threaded 34-mm lateral mass screws were inserted bilaterally. The posterior arch fracture was reduced under direct vision by approximating the lateral mass screw heads. A rod was then cut to measure, contoured, and fitted to the screws, achieving further reduction of the anterior arch. End caps were then tightened to achieve compression across the fracture (Figures 3 and 4). The wound was finally washed with saline and a radivac drain 


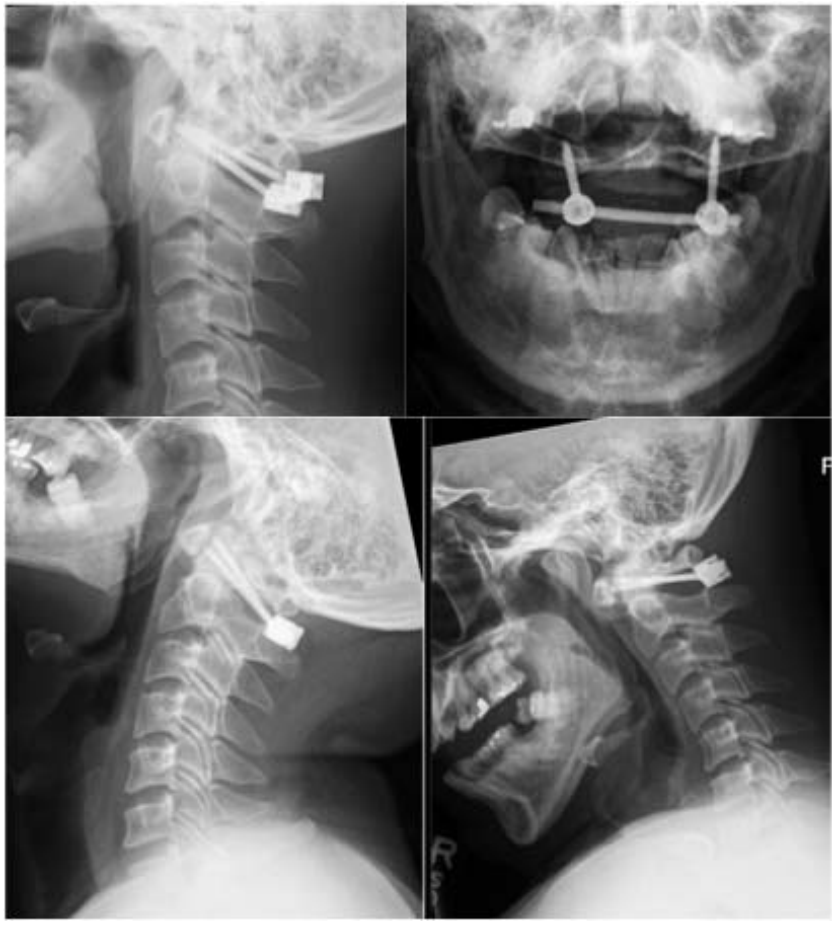

Figure 4. Postoperative radiographs demonstrating the stability of the construct.

inserted before executing a layered closure. An Aspen collar was applied postprocedure.

The patient made an uneventful recovery and was discharged 2 days later. The collar was worn for 6 weeks before weaning off over the subsequent 6 weeks. Follow-up radiographs were performed at 1 and 3 months along with CT scan at 3 months. At 3 months, he demonstrated near full range of motion of the cervical spine, was pain free, and had resumed driving (Figure 5). At 6 months, he returned to work as an airline steward. At 12 months, CT scan

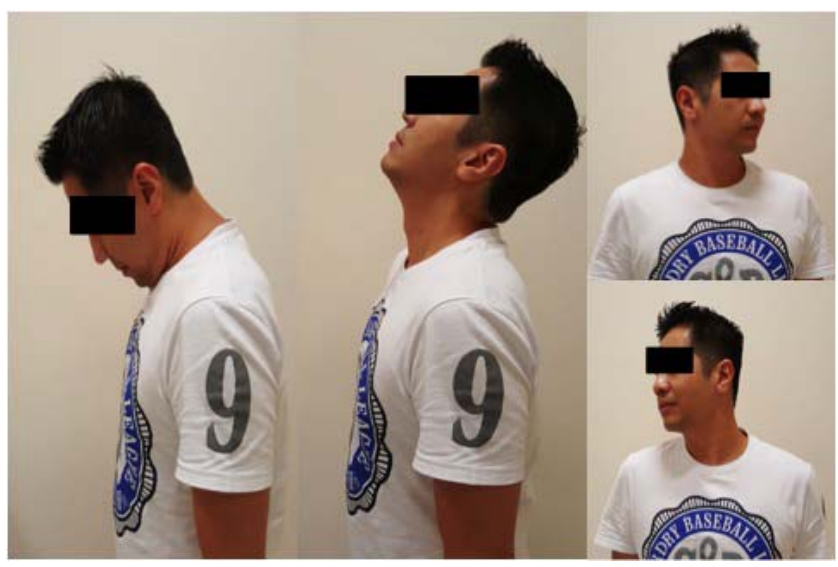

Figure 5. Patient showing near normal range of flexion, extension, and rotation of the cervical spine at 3 months postsurgery.

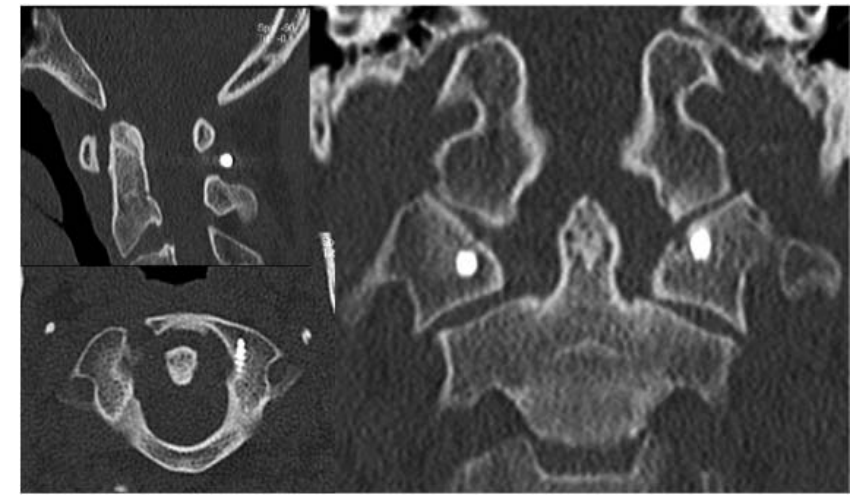

Figure 6. Computed tomographic scan at 1 year postoperation showing maintenance of reduction, bony union of the posterior arch, and fibrous union of the anterior arch of $\mathrm{C} 1$.

confirmed union (Figure 6). Clinically, the patient experienced slight discomfort after prolonged sitting or standing but remained painless through a full range of movement.

\section{DISCUSSION}

Neck pain is the most common presentation of Jefferson fractures. Neurological injuries are uncommon since the large diameter of the spinal canal is further widened by centrifugal displacement of fragments. ${ }^{4}$ Standard radiographs of occipito-cervical junction are often inadequate and overlook these fractures. Hence, a high index of suspicion must be maintained in patients with neck pain who have sustained axial compression injuries.

The integrity of the transverse ligament determines the stability of Jefferson fractures. ${ }^{1}$ Transverse ligament injuries are classified into intrasubstance tears (type I) or bony avulsion fractures of the ligament from $\mathrm{C} 1$ lateral mass (type II). Type II injuries show a promising $74 \%$ healing rate with conservative management, while type I injuries exhibit poor healing rates that warrant surgical intervention. ${ }^{5}$ An atlanto-dens interval $>3$ $\mathrm{mm}$ or cumulative lateral mass displacement $>8.1$ $\mathrm{mm}$ on open mouth odontoid view radiographs imply rupture of the transverse ligament and hence fracture instability ${ }^{6}$ (Figure 1).

Our case is an unstable type III atlas fracture involving both anterior and posterior arches with associated intrasubstance transverse ligament injury. It is cited to have a $33.3 \%$ prevalence of nonunion across both operative and nonoperative management. ${ }^{7}$ Other atlas fracture types and their associated nonunion prevalence are detailed in 
Table 1. Types of Jefferson burst fractures.

\begin{tabular}{|c|c|c|c|}
\hline Classification of Fractures & Mechanism of Injury & Diagnostic Findings & Nonunion Prevalence $(\%)^{\mathrm{a}}$ \\
\hline Type I & $\begin{array}{l}\text { Axial load and flexion or } \\
\text { extension }\end{array}$ & $\begin{array}{l}\text { Isolated fracture of anterior or posterior } \\
\text { arch }\end{array}$ & $4.8^{\mathrm{a}}$ \\
\hline Type II & Axial load & $\begin{array}{l}\text { Bilateral fractures of anterior and } \\
\text { posterior arch }\end{array}$ & $13.6^{\mathrm{a}}$ \\
\hline Type III & Axial load and rotation & Lateral mass fracture & $33.3^{\mathrm{a}}$ \\
\hline Atlanto-occipital dislocation & $\begin{array}{l}\text { Distraction and hyperextension or } \\
\text { hyperflexion of atlanto-occipital } \\
\text { joint }\end{array}$ & $\begin{array}{l}\text { Severe disruption of ligaments between } \\
\text { base of skull and atlas }\end{array}$ & Not reported \\
\hline
\end{tabular}

${ }^{\mathrm{a}}$ Lleu et al. ${ }^{7}$

Table 1. The management of unstable Jefferson fractures is controversial. HALO immobilization is a viable option with acceptable radiographic results and low rates of nonunion or instability. ${ }^{8}$ However, the patient rejected it because of the perceived practical difficulties and inconvenience of managing it for a period of potentially up to 3 months. The 1week delay in presentation was also taken into consideration as a risk factor for nonunion with conservative measures, thus further justifying the use of direct operative fixation. ${ }^{8}$

Unstable Jefferson fractures are traditionally treated with fusion. Occipito-cervical fusion is helpful when purchase of $\mathrm{C} 1$ is inadequate. Unfortunately, fusions cause significant loss of range of motion, up to $52^{\circ}$ in the flexion-extension plane and $38.9^{\circ}$ in rotation, ${ }^{9}$ making it particularly disabling for younger patients. C1-C2 posterior fusion, though less extensive, can be challenging if the $\mathrm{C} 1$ C2 joint is not reduced. McGuire and Harkey ${ }^{10}$ proposed executing it with transfacet $\mathrm{C} 1-\mathrm{C} 2$ screw fixation to preserve motion at the occipital cervical level, but this sacrifices C1-C2 movement.

The technique employed here was direct fixation of $\mathrm{C} 1$ with a posterior approach. Although described before by Li et $\mathrm{al}^{3}$ and Bransford et $\mathrm{al}^{2}$ to have positive functional outcomes, it is still not widely practiced. This approach affords better exposure and provides the option of stabilizing adjacent vertebrae if necessary. The idea is to obtain stable fixation that enables the transverse ligament to heal and achieve stable fibrous union while preserving cervical spine range of motion. While no major complications have yet to be reported, the advent of surgical navigation would further minimize the risk of injuring surrounding structures with a more precise placement of screw and a less invasive approach. ${ }^{11}$

While this technique has been described before, it is still rarely utilized outside major institutions, and we hope to raise awareness of this simple technique that allows for earlier return to work and social life. We believe that using monoaxial screws along with contoured rods would allow us to reduce the anterior arch fracture more effectively, but these screws are currently not available to us.

\section{REFERENCES}

1. Ryken TC, Aarabi B, Dhall SS, Gelb DE, Hurlbert RJ, Rozzelle CJ, et al. Management of isolated fractures of the atlas in adults. Neurosurgery. 2013;72(suppl 2):127-131.

2. Bransford R, Chapman JR, Bellabarba C. Primary internal fixation of unilateral $\mathrm{C} 1$ lateral mass sagittal split fractures: a series of 3 cases. $J$ Spinal Disord Tech. 2011;24(3):157-163.

3. Li L, Teng H, Pan J, Qian L, Zeng C, Sun G, et al. Direct posterior cl lateral mass screws compression reduction and osteosynthesis in the treatment of unstable jefferson fractures. Spine (Phila Pa 1976). 2011;36(15):E1046-E1051.

4. Hu Y, Yuan ZS, Kepler CK, Dong WX, Sun XY, Zhang J. Comparison of occipitocervical and atlantoaxial fusion in treatment of unstable Jefferson fractures. Indian J Orthop. 2017;51(1):28-35.

5. Dickman CA, Greene KA, Sonntag VK. Injuries involving the transverse atlantal ligament: classification and treatment guidelines based upon experience with 39 injuries. Neurosurgery. 1996;38(1):44-50.

6. Heller JG, Viroslav S, Hudson T. Jefferson fractures: the role of magnification artifact in assessing transverse ligament integrity. J Spinal Disord. 1993;6(5):392-396.

7. Lleu M, Charles YP, Blondel B, et al. C1 fracture: analysis of consolidation and complications rates in a prospective multicenter series. Orthop Traumatol Surg Res. 2018;104(7):1049-1054.

8. Zura R, Mehta S, Della Rocca GJ, Steen RG. Biological risk factors for nonunion of bone fracture. JBJS Rev. 2016;4(1):XXX-XXX.

9. Panjabi M, Dvorak J, Crisco J III, Oda T, Hilibrand A, Grob D. Flexion, extension, and lateral bending of the upper cervical spine in response to alar ligament transections. $J$ Spinal Disord. 1991;4(2):157-167.

10. McGuire RA Jr, Harkey HL. Primary treatment of unstable Jefferson's fractures. J Spinal Disord. 1995;8(3):233236. 
11. Mezger U, Jendrewski C, Bartels M. Navigation in surgery. Langenbeck's Arch Surg. 2013;398(4):501-514.

Disclosures and COI: This is to declare that there are no conflicts of interest and source of funding in the writing of this case report.

Corresponding Author: Aravind Kumar, MBBS, MRCS(Ed), MRCS(Glas), FRCS, FAMS, $\mathrm{Ng}$ Teng Fong Hospital, 1 Jurong East Street 21,
Singapore 609606. Phone: +65 91246131; Fax: +65 67165500; Email: draravind@gmail.com.

Published 31 August 2019

This manuscript is generously published free of charge by ISASS, the International Society for the Advancement of Spine Surgery. Copyright (C) 2019 ISASS. To see more or order reprints or permissions, see http://ijssurgery.com. 